\title{
Pulmonary Organs Analysis Method and Its Evaluation Based on Thoracic Thin-Section CT Images
}

\author{
Tetsuya Tozaki ${ }^{1}$, Akira Tanaka ${ }^{2}$, Yoshiki Kawata ${ }^{2}$, Noboru Niki $^{2}$, \\ Hironobu Ohmatsu ${ }^{3}$, Ryutaro Kakinuma ${ }^{3}$, Kenji Eguchi ${ }^{4}$, \\ Masahiro Kaneko ${ }^{5}$, and Noriyuki Moriyama ${ }^{5}$ \\ 1 Dept. of Electronic Eng., Kobe City College of Tech. \\ 8-3 Gakuen-higashi-machi, Nishiku, Kobe 651-2194, JAPAN \\ tozaki@kobe-kosen.ac.jp \\ 2 Dept. of Optical Science, Univ. of Tokushima \\ 2-1 Minami-josanjima-cho, Tokushima 770-8560, JAPAN \\ \{atanaka, niki, kawata\}@opt. tokushima-u.ac.jp \\ 3 National Cancer Center Hospital East \\ 4 National Shikoku Cancer Center Hospital \\ ${ }^{5}$ National Cancer Center Hospital
}

\begin{abstract}
To diagnose the lung cancer as to determine if it has malignant or benign nature, it is important to understand the spatial relationship among the abnormal nodule and other pulmonary organs. But the lung field has very complicated structure, so it is difficult to understand the connectivity of the pulmonary organs using thin-section CT images. This method consists of two parts. The first is the classification of the pulmonary structure based on the anatomical information. The second is the quantitative analysis that is then applicable to differential diagnosis, such as differentiation of malignant or benign abnormal tissue.
\end{abstract}

\section{Introduction}

The lung area has very complicated structure, so it is difficult to understand the spatial relationships among the pulmonary organs and the abnormal nodules. Because of this, a new Computer Assisted Diagnosis system for lung cancer becomes necessary [1].

In this paper, we describe a 3D analysis method of the lung based on thinsection CT images, and we aim to support the differential diagnosis of lung cancer. Our pulmonary organs analysis method consists of four parts. The first is the extraction of the regions of interest (ROI) based on the segmentation of the lung field. The second is the division of ROI into the bronchus and the blood vessels, and the third is the identification of the pulmonary artery and the pulmonary vein using anatomical information and 3D image processing technology $[2,3]$. The last is a quantitative analysis of the spatial relationships among the abnormal nodules and other pulmonary organs to support the differential diagnosis of lung cancer, such as differentiation of malignant or benign nodules. 


\section{Extraction of the Bronchus, the Pulmonary Artery, and the Pulmonary Vein}

\subsection{Bias Correction}

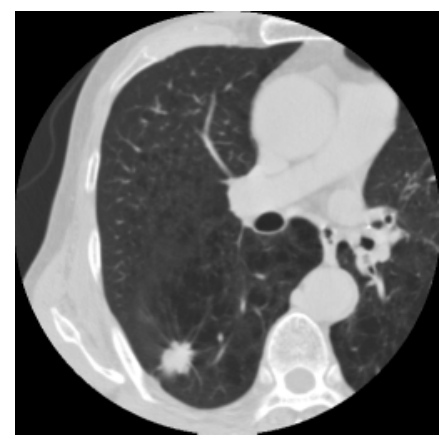

(a) thin-section CT image

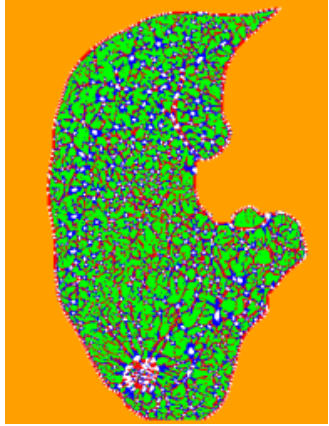

(b) Intensity curve segmentation

Fig. 1. Thin-section CT image and the result of intensity curve segmentation. (a) shows a thin-section CT image, (b) shows a result of intensity curve segmentation against (a).

Fig.1 (a) shows a thin-section CT image at measurement condition, tube voltage, $150 \mathrm{kV}$, tube current $200 \mathrm{~mA}$, beam width $2 \mathrm{~mm}$, table speed $2 \mathrm{~mm} / \mathrm{s}$. Thin-section CT images have a large smooth bias component caused by various artifacts or diseases. To get high quality ROI images, we must remove this bias component. The correction method of the bias component is based on the segmentation of the lung field using Gaussian curvature and mean curvature. Calculation of Gaussian curvature $K$ and mean curvature $H$ are expressed in Eqs.(1) and (2) [4,5].

$$
\begin{gathered}
K=\frac{1}{2 h^{2}}\left(f_{x x} f_{y y}+f_{x y} f_{y x}\right) \\
H=\frac{1}{2 h^{\frac{3}{2}}}\left(f_{x x}-2 f_{x} f_{x y} f_{y}+f_{x x} f_{y}^{2}+f_{y y}+f_{x}^{2} f_{y y}\right)
\end{gathered}
$$

where $f$ is the smoothed image by Gaussian filter, $h=1.0+f_{x}{ }^{2}+f_{y}{ }^{2}$ and subscripts denote partial differentiation at the $(x, y)$.

We segment the lung field into four regions based on the combination of the sign of two curvature. Each region express features such as pit surface $(K>0$ and $H>0)$, peak surface $(K>0$ and $H<0)$, saddle ridge $(K<0$ and $H<0)$, saddle valley $(K<0$ and $H>0)$ [6]. Fig.1 (b) shows a result of the intensity curve segmentation. In this figure, white regions are peak surface, green regions are pit surface, blue regions are saddle ridge, and red regions are saddle valley. We separate the segmented image into background regions and the ROI. Background regions are expressed by pit surfaces because of beam hardening effect, and ROI 
are expressed by peak surface, saddle ridge, and saddle valley. We approximate the bias component with a bicubic spline function, and the control points are on pit surface region. Then we subtract these estimated bias field from the original thin-section CT images.

\subsection{Extraction of ROI}

Peak surface, saddle ridge, and saddle valley are mostly consisted of the bronchus, the pulmonary artery, and the pulmonary vein. But these regions also include background of lung field near the boundary of ROI. To extract only ROI, we apply thresholding technique and edge emphasizing to bias corrected image. Core of ROI have relatively high $\mathrm{CT}$ value than edge, so we extract these region by thresholding technique. We use edge emphasizing to extract peripheral ROI. As the edge emphasizing filter, we apply Marr-Hildreth operator. We extract the edge of these regions by zero-crossing method [7].

\subsection{Bronchus and Blood Vessels}

The bronchus is described as a tree-like tube filled with air. CT values inside the tube are almost equal to the low $\mathrm{CT}$ values of air and $\mathrm{CT}$ values of the bronchus wall are as high as the blood vessels. The difference of CT values among the bronchus wall, the pulmonary artery, and the pulmonary vein is not distinctable. We present an algorithm to differentiate the bronchus based on the extraction of the air region inside the bronchus.

To get a high quality image of the bronchus and the blood vessels, we must extract the air regions inside the peripheral bronchus. We cannot, however, extract these air regions inside the peripheral bronchus by a simple thresholding algorithm because these parts have higher $\mathrm{CT}$ values than in the trunk bronchus. This is due to artifacts such as the partial volume effect. We extract the air region inside peripheral bronchus using the following procedures [8].

Step 1) Extraction of the initial air region based on threshold technique.

Step 2) Growth initial air region.

Step 3) Extraction of the air region inside the peripheral bronchus using intensity curve segmentation information.

In step 1), we use the extraction method described by K.Mori et al.. [9]. In Step 2), we emphasize the edge of air region using Laplacian Operator, and grow the initial air region obtained by Step 1) until it correspond to edge using region growing method morphologically. In Step 3), we use the intensity curve segmentation information to get the air region inside peripheral bronchus. On Thin-section CT images, the peripheral bronchus is expressed as a ring shape. In this case, inside the ring shape becomes an isolated small Pit Surface region through the intensity curve segmentation method that has smoothing process by small filter size. It is based on the fact that the inside of the peripheral bronchus has a pit feature in small region on the intensity curve field. We extract these pit Surface regions which connect to the air region obtained by Step 2) as the air regions inside peripheral bronchus. 


\subsection{Extraction of the Pulmonary Artery and the Pulmonary Vein}

The pulmonary artery runs along the bronchus. The identification procedure of the pulmonary artery and the pulmonary vein is as follows [8].

Step 1) We obtain the initial blood vessels image at relatively high CT values from pulmonary blood vessels.

Step 2) We obtain centerline structures of the initial blood vessels using a 3D thinning algorithm [10].

Step 3) We represent the centerline structures as the set of branches.

Step 4) We classify the branches of the initial blood vessels into two classes based on the running direction of the branches against the bronchus.

Step 5) We identify blood vessel to the pulmonary artery and the pulmonary vein based on classified centerline structure using spatial connectivity of the blood vessels.

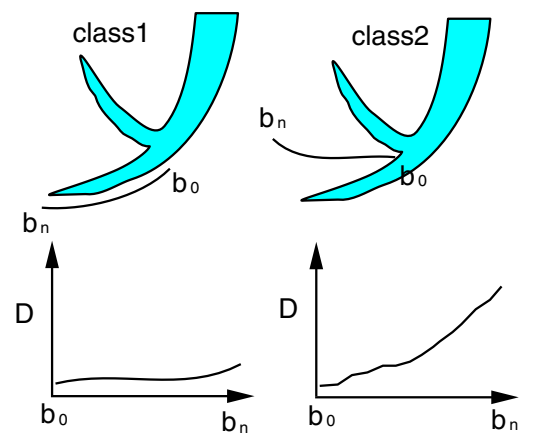

Fig. 2. Classification criterion

In Step 1), we extract the 3D main structure of blood vessels to keep the skeleton information of pulmonary blood vessels, and remove contact parts among the peripheral pulmonary artery and the peripheral pulmonary vein.

In Step3), we remove small branches caused by the irregular shape of the blood vessels, and we represent the centerline structure as the set of branches after removing the branching points of centerline structures.

In Step 4), we calculate the relationship among blood vessels branches and bronchus, and we classify the blood vessel branches into two classes. To represent the relationship among blood vessels and bronchus, we use the graph pattern as shown in Fig.2. In this figure, horizontal axis shows the sequential voxel number from the edge of the interesting branches, and vertical axis shows the minimum spatial distance $D_{i}$ from the interesting voxel to the bronchus. If this graph has little change of spatial distance $D_{i}$ and the value of $D_{i}$ is lower than $T$, this branch is classified as pulmonary artery, otherwise if the value of $D_{i}$ increases or decreases with the change of the interesting point, this branch is classified as pulmonary vein. Based on these graph pattern, we classify blood vessels branches into pulmonary artery or pulmonary vein. 


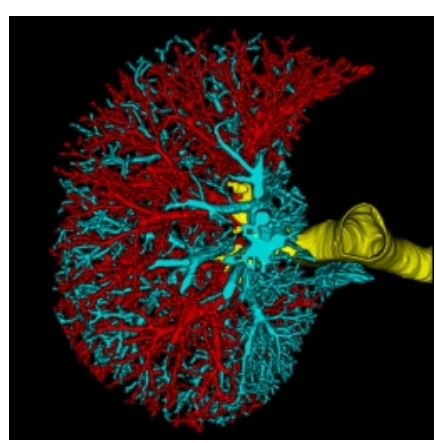

(a) normal case

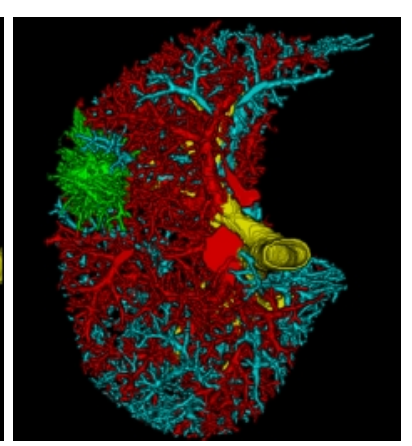

(b) lung cancer case

Fig. 3. Classified lung organs

Fig.3(a) and (b) shows the whole pulmonary organs image consisted of the bronchus, the pulmonary artery, the pulmonary vein and the tumor. (a) shows the analysis result of normal lung, and (b) shows the lung cancer case. Red organ are the pulmonary artery, blue are the pulmonary vein, yellow are the bronchus, and green are the abnormal nodule extracted by interactive operation.

\section{Quantitative Analysis}

From the classified pulmonary organs images, we make quantitative analysis for differential diagnosis of lung cancer against follows items to support determination such as malignant or benign nature.

1) The number of connections among the pulmonary blood vessels and the abnormal nodule. 2) The direction of the blood vessels which connect to the abnormal nodule.

As the typical feature of malignant lung cancer, we can observe that the pulmonary blood vessels around the tumor are drawn in irregularly by the lung cancer tissue. We represent these malignant features using centerline of blood vessels obtained by $3 \mathrm{D}$ thinning algorithm. Understanding of the connection number is operated as follows. Firstly, we extract the abnormal nodule and the pulmonary blood vessels that connect to this abnormal nodule. Next, we apply a $3 \mathrm{D}$ thinning algorithm only to pulmonary vessels. Then we count the connection of the abnormal nodule and the centerline of the blood vessels each pulmonary artery and pulmonary vein.

The direction of the blood vessels that connect to the abnormal nodule is calculated as follows. Now the unit direction vector of the interesting blood vessels branch is $\hat{d}_{P}$ which is represented by the tangent direction at the connection point, and the unit direction vector from the connection point $\mathrm{P}$ to the center point of the abnormal nodule $\mathrm{Q}$ is $\hat{d}_{Q}$. Then we define the direction of the blood vessels that connect to the abnormal nodule as $V=\hat{d}_{P} \cdot \hat{d}_{Q}$ If $\mathrm{V}$ is larger than 0.9 , we guess this branch are drawn in by the abnormal nodule irregularly. 
Table1 (a) shows the result of the quantitative analysis against four malignant lung cancer cases, and (b) shows the result against four benign cases. Columns of each case show the connection number of the final pulmonary blood vessels. These columns show the complexity of state among these organs. A numerator shows the number of the blood vessels which head to the center of the abnormal nodule, a denominator shows the number of the blood vessels that connect to the abnormal nodule. "P.V." means the pulmonary vein connection. "P.A." means the pulmonary artery connection. We can understand there are obvious difference among malignant and benign cases. In malignant cases, the numbers of the blood vessels that head to center of the abnormal nodule are very large. And further, graph 1,2,3 understand there are more obvious difference among malignant and benign cases. Graph 1,2,3 show blood vessels connection in malignant cases is larger than that in benign cases, and show the ratio of the blood vessels which head to center of the abnormal nodule are very large.

Table 1. Application of the quantitative analysis.

(a) malignant cases

(b) benign cases

\begin{tabular}{|c||c|c|c|c|c||c|c|c|c|}
\hline Cases & P.V. & P.A. & Total & ratio & Cases & P.V. & P.A. & Total & ratio \\
\hline Case1 & $4 / 4$ & $2 / 5$ & $6 / 9$ & 0.667 & Case1 & $2 / 7$ & $0 / 2$ & $2 / 9$ & 0.222 \\
\hline Case2 & $8 / 12$ & $3 / 5$ & $11 / 17$ & 0.647 & Case2 & $2 / 3$ & $0 / 1$ & $2 / 4$ & 0.5 \\
\hline Case3 & $3 / 8$ & $3 / 13$ & $6 / 21$ & 0.286 & Case3 & $1 / 3$ & $1 / 2$ & $2 / 5$ & 0.4 \\
\hline Case4 & $9 / 13$ & $10 / 17$ & $19 / 30$ & 0.633 & Case4 & $1 / 10$ & $3 / 13$ & $4 / 23$ & 0.174 \\
\hline
\end{tabular}

graph 1

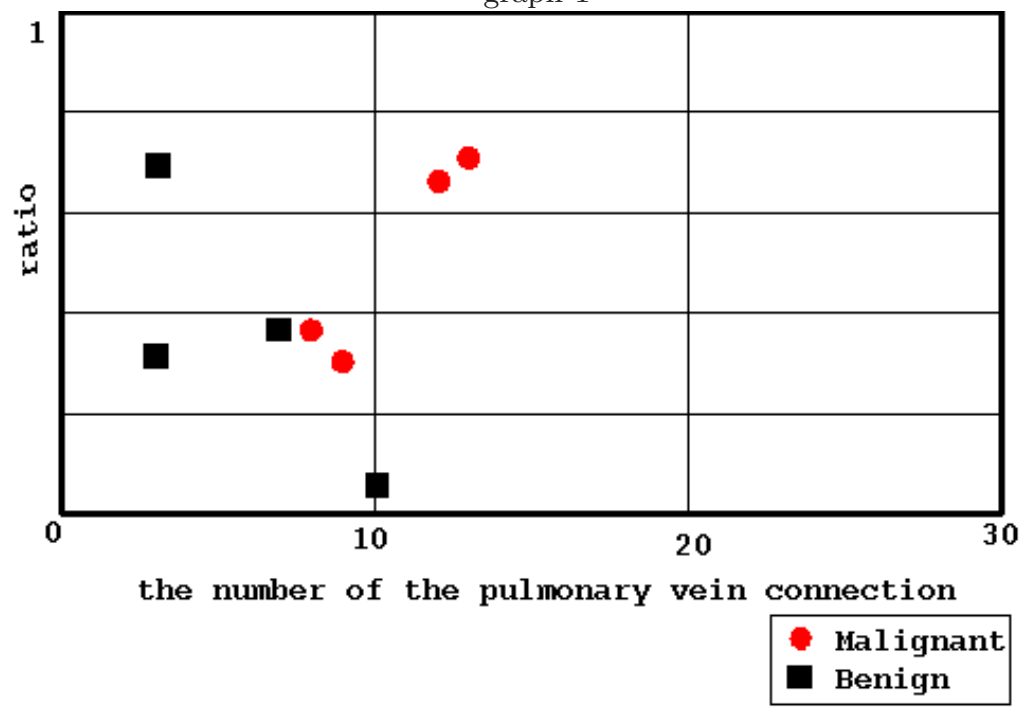


graph 2

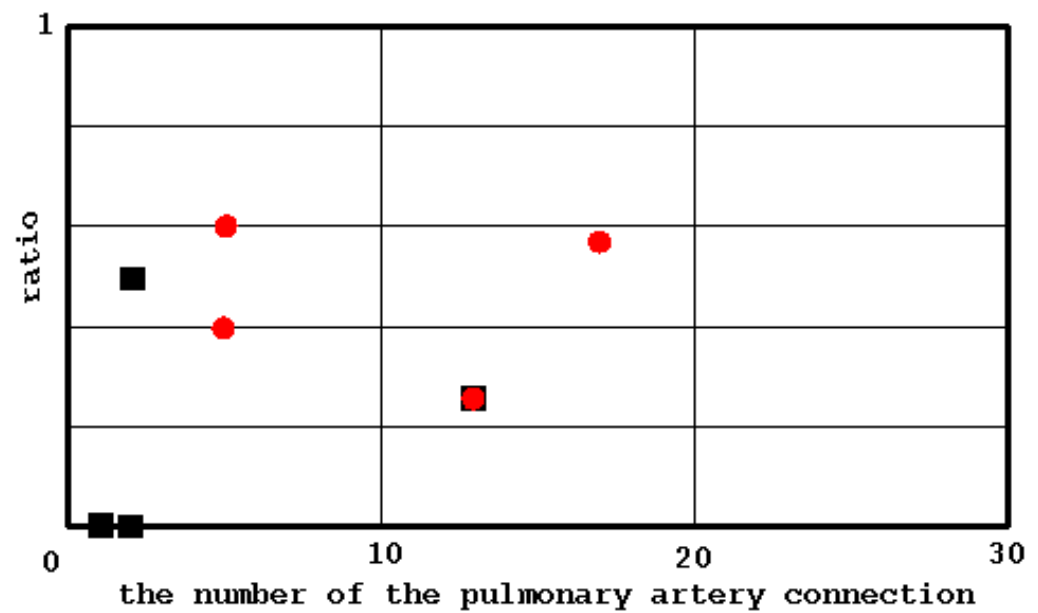

Malignant

Benign

graph 3

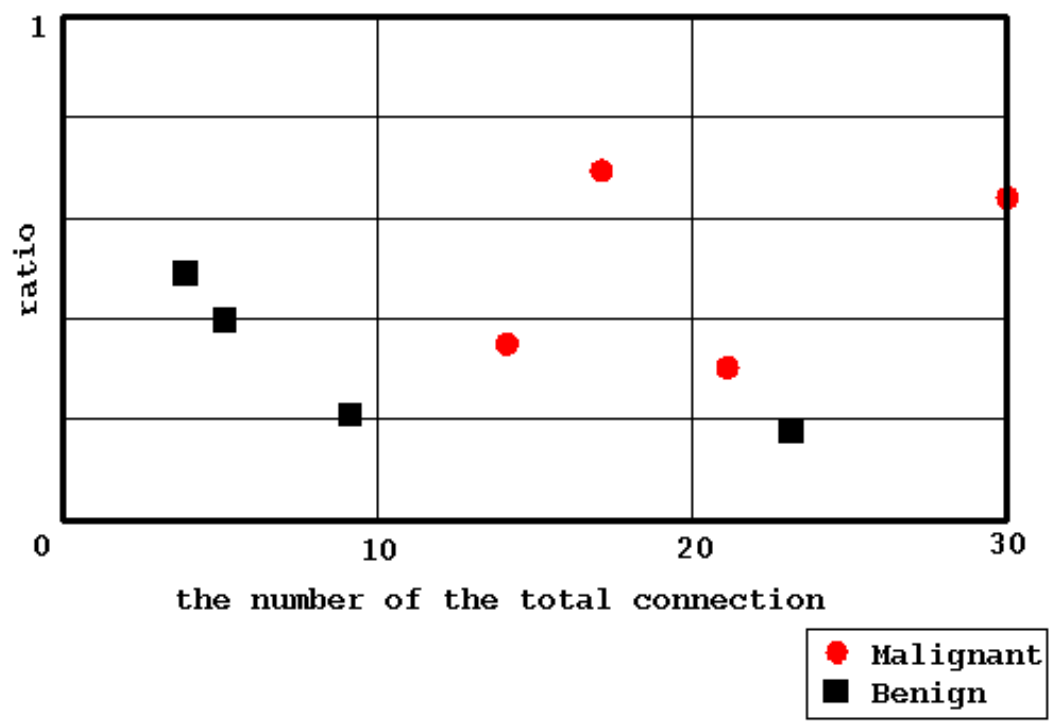

\section{Conclusion}

In this paper, we described a 3D image analysis method of the pulmonary organs structure based on thin-section CT images, and we applied this method to 
some normal lung and patient cases. By the segmentation of the lung filed using Gaussian curvature and mean curvature, we can extract peripheral ROI. Using anatomical informations of lung, we can classify the pulmonary organs to the bronchus, the pulmonary artery, and the pulmonary vein. We also applied these analysis results to a differential diagnosis of lung cancer. We could understand the spatial connectivity among the pulmonary organs and the abnormal nodules easily. A quantitative analysis of the relationship between the pulmonary organs and the abnormal nodules, can be expected to support differential diagnosis for a malignant or benign decision on the abnormal nodule. In future work, we will extract the bronchus, the pulmonary artery, and the pulmonary vein using more detailed anatomical information, and we will develop this image analysis method to support the differential diagnosis of lung cancer in the clinical medicine.

\section{References}

1. K. Kanazawa, M. Kubo, N. Niki, H.Satoh, H.Ohmatsu, K.Eguchi, N. Moriyama, "Computer Aided Screening System for Lung Cancer Based on Helical CT Images", proc. 4th VBC, Hamburg, vol.1131, pp.223-228, 1996. 394

2. T.Tozaki, Y.Kawata, N.Niki, H.Ohmatsu, K.Eguchi, N.Moriyama, "Three dimensional analysis of lung areas using thin slice CT images", SPIE Medical Imaging, Vol.2709, pp.2-11, 1996. 394

3. T. Tozaki, Y. Kawata, N. Niki, H.Ohmatsu, R.Kakinuma, K. Eguchi, N. Moriyama, "Pulmonary Organs Analysis for Differential Diagnosis Based on Thoracic Thinsection CT Images", IEEE Trans. on NUCLEAR SCIENCE, vol.45, pp.3075-3082, NO.6, 1998. 394

4. R.Deriche, "Recursively Implementing the Gaussian and Its Derivatives", In Proc. Second Int. Conf. On Image Processing, pp.263-267, 1992. 395

5. O. Monga, R. Lengagne, R. Deriche, "Crest lines extraction in volume 3D medical images: a multi-scale approach", INRIA RR2338, 1994. 395

6. Y.Kawata, N.Niki, T.Kumazaki, "Feature Extraction of Convex Surface on Blood Vessels Using Cone-Beam Images", IEEE International Conference on Image Processing, Vol.3, pp.315-318, 1996. 395

7. D. Marr, E. Hildreth, "Theory of edge detection", In Processing of the Royal Society of London, pp.187-217, 1980. 396

8. T.Tozaki, Y.Kawata, N.Niki, H.Ohmatsu, R.Kakinuma, K.Eguchi, M.Kaneko, N.Moriyama, "Pulmonary organs analysis method and its application to differential diagnosis based on thoracic Thin-section CT images", SPIE Medical Imaging, Vol.3338, pp.1459-1469. San Diego, 1998. 396, 397

9. K.Mori, J.Hasegawa, J.Toriwaki, H.Anno, K.Katada, "Recognition of Bronchus in Three-Dimensional X-ray CT Images with Application to Virtualized Bronchoscopy System", proc. 13th ICPR, vol. III, Track C, pp528-532, Vienna, 1996. 396

10. N.Niki, Y.Kawata, T.Kumazaki, "3D Diagnostic Imaging of Blood Vessels Using an X-Ray Rotational Angiographic System", AAAI 1994 spring symposium, Stanford University, pp.169-172, 1994. 397 